\title{
Slow Atomic Motion in Zr-Ti-Cu-Ni-Be Metallic Glasses Studied by NMR
}

\author{
X.-P. Tang, ${ }^{1}$ Ralf Busch, ${ }^{2}$ William L. Johnson, ${ }^{2}$ and Y. $\mathrm{Wu}^{1, *}$ \\ ${ }^{1}$ Department of Physics and Astronomy, University of North Carolina, Chapel Hill, North Carolina 27599-3255 \\ ${ }^{2}$ Keck Laboratory of Engineering Materials, California Institute of Technology, Pasadena, California 91125
}

(Received 1 June 1998)

\begin{abstract}
Nuclear magnetic resonance is used for the first time to detect slow atomic motion in metallic glasses, specifically, Be motion in $\mathrm{Zr}-\mathrm{Ti}-\mathrm{Cu}-\mathrm{Ni}-\mathrm{Be}$ bulk metallic glasses. The observations are not consistent with the vacancy-assisted and interstitial diffusion mechanisms and favor the spread-out free volume fluctuation mechanism for Be diffusion. Comparison with the results of Be diffusion measured by elastic backscattering the NMR results also indicates that the energy barriers for short- and long-range Be motion are the same. [S0031-9007(98)07917-4]
\end{abstract}

PACS numbers: 66.30.Fq, 07.57.Pt, 61.18.Fs, 61.43.Dq

The nature of atomic transport in metallic glasses has been the subject of numerous studies over the past decade and still remains a controversial and highly debated issue. Traditional techniques employed for diffusion study in metallic glasses include radioactive tracer [1,2], secondary ion mass spectrometry [3-6], and elastic backscattering (EBS) [7]. These techniques are based on the analysis of long-range diffusion and do not probe directly the characteristics of short-range atomic motions. For instance, possible spatial inhomogeneity of atomic motions cannot be probed directly by these techniques [1]. In contrast, nuclear magnetic resonance (NMR) is a direct probe of local atomic motions and has made significant contributions to the understanding of motions in polymeric and oxide glassy systems [8-12] and in crystalline metals and alloys [13-16]. So far, the potential of NMR has not been realized in studying atomic motions in metallic glasses [12].

Recently, bulk metallic glasses $\mathrm{Zr}_{41.2} \mathrm{Ti}_{13.8} \mathrm{Cu}_{12.5} \mathrm{Ni}_{10^{-}}$ $\mathrm{Be}_{22.5}$ (vit1) and $\mathrm{Zr}_{46.75} \mathrm{Ti}_{8.25} \mathrm{Cu}_{7.5} \mathrm{Ni}_{10} \mathrm{Be}_{27.5}$ (vit4) with extraordinarily high glass forming ability and thermal stability were discovered $[17,18]$. The Be diffusivity in both glasses was found by EBS to follow Arrhenius behavior below $T_{g}(\approx 625 \mathrm{~K})$ with activation enthalpy of $1.1 \mathrm{eV}$ [7]. Because of the lack of a convenient Be isotope, these EBS studies measure the diffusion profile of $\mathrm{Be}$ atoms from deposited Be surface layers on vit1 and vit4. The NMR investigation of Be motion does not involve $\mathrm{Be}$ concentration gradient. Using the ${ }^{9} \mathrm{Be}$ spin alignment echo technique $[8,9,19]$, the characteristics of slow motions with Be jump frequency as low as 1 jump per $10 \mathrm{sec}$ are investigated. Direct experimental evidences are provided indicating that Be motions are not consistent with vacancyassisted diffusion and interstitial diffusion. The result is explained in terms of the thermal fluctuation of spreadout free volume which is consistent with the measured negative effective entropy. Beryllium motions detected by both NMR and EBS follow Arrhenius behavior with the same activation enthalpy. This demonstrates that the energy barrier for short-range Be motion is the same as that for long-range diffusion in vit1 and vit4.
The preparation of vit 1 and vit 4 ingots has been described elsewhere [17]. The ingots were cut into thin slices of $200 \mu \mathrm{m}$ thickness and vacuum sealed in glass tubes for NMR measurements. A home-built high temperature probe was used to conduct NMR experiments on a pulsed spectrometer at 9.4 T. Spectra were also obtained at $4.7 \mathrm{~T}$ to verify the nature of the line broadening. In this work the $90^{\circ} \mathrm{rf}$ pulse is about $4 \mu \mathrm{s}$ which ensures nonselective excitations of both the central and satellite transitions.

Features of ${ }^{9} \mathrm{Be}$ spectra are the same for vit 1 and vit 4 . In the entire temperature range of the current investigation there is no noticeable change of the spectrum. Figure 1 shows the room temperature ${ }^{9} \mathrm{Be}$ spectrum of vit1 at $9.4 \mathrm{~T}$ obtained by the quadrupole echo pulse sequence. The spectrum consists of two components. The full width at half height $(\mathrm{FWHH})$ of the broad line is about $(100 \pm$ 10) $\mathrm{kHz}$ which remains the same at $4.7 \mathrm{~T}$. Such field independent linewidth indicates that the broad line is associated with the satellite transitions broadened by the

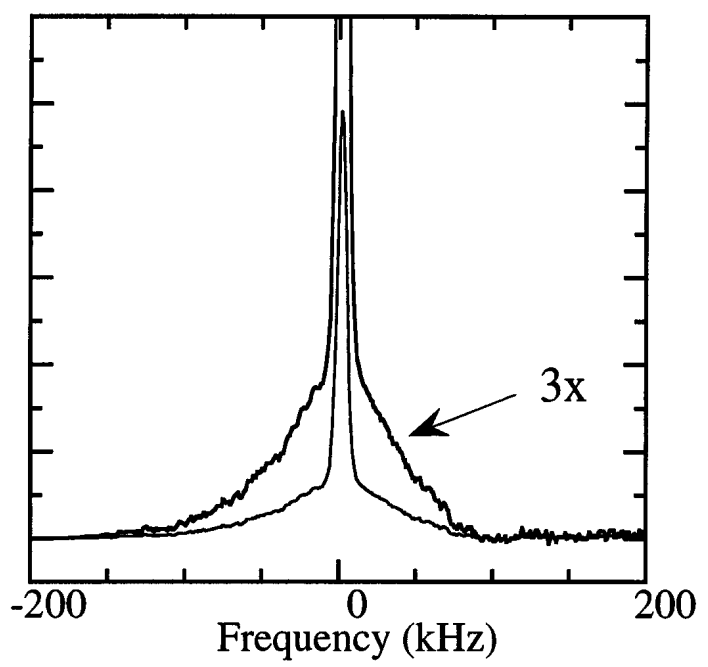

FIG. 1. The ${ }^{9} \mathrm{Be}$ spectrum of vit1 at $9.4 \mathrm{~T}$. The satellite transition is shown more clearly by expanding the vertical scale by a factor of 3 . 
first-order quadrupole interactions. The FWHH of the central peak is $(6.1 \pm 0.2) \mathrm{kHz}$ (about $110 \mathrm{ppm}$ ) and is reduced to $(3.6 \pm 0.1) \mathrm{kHz}$ at $4.7 \mathrm{~T}$. Thus, the central peak is mainly broadened by the Zeeman interaction distribution. The intensity ratio of the narrow line to the broad line is approximately 4:6 which is the expected intensity ratio of the central transition to the satellite transitions for spin$3 / 2$ nuclei. The characteristic quadrupole powder pattern of ${ }^{9} \mathrm{Be}$ spectra is smeared out in vit 1 and vit4 in contrast to the characteristic powder pattern of ${ }^{9} \mathrm{Be}$ spectrum observed in pure $\mathrm{Be}$ metal [20]; the local environment around $\mathrm{Be}$ is the same from site to site in Be metal, whereas the truncated quadrupole interaction in glasses is expected to be different from site to site.

The Knight shift in vit 1 and vit 4 is about 0 ppm. The shift reference is obtained through the ${ }^{2} \mathrm{H}$ resonance frequency in $\mathrm{D}_{2} \mathrm{O}$ and the ratio of ${ }^{9} \mathrm{Be}$ and ${ }^{2} \mathrm{H}$ gyromagnetic ratios [20]. The ${ }^{9} \mathrm{Be}$ spin-lattice relaxation time $T_{1}$ is obtained by the saturation recovery method. Figure 2 shows the nuclear magnetization $M(t)$ as a function of the recovery time $t\left[M^{*}(t) \equiv[M(\infty)-M(t)] / M(\infty)\right]$ at $300 \mathrm{~K}$. It can be fitted perfectly with a single exponential function $M^{*}(t)=\exp \left(-t / T_{1}\right)$ with $T_{1}=3.55 \mathrm{~s}$. The inset of Fig. 2 shows $1 / T_{1}$ vs $T$. In both glasses, $1 / T_{1}$ is approximately proportional to $T$ with $\left(T T_{1}\right)^{-1}=$ $0.00105 \mathrm{~K}^{-1} \mathrm{~s}^{-1}$ up to $625 \mathrm{~K}$. Thus, the ${ }^{9} \mathrm{Be} T_{1}$ is dominated by the coupling with conduction electron spins [21]. No effects of phonons and fast atomic motions are visible in the $T_{1}$ data. The ${ }^{9} \mathrm{Be}$ spin-spin relaxation time $T_{2}$ measured by the quadrupole echo pulse sequence is about $1.5 \mathrm{~ms}$ and is temperature independent. The temperature independent $T_{2}$ and line shape indicate the absence of atomic motions with time scales shorter than $1 \mathrm{~ms}$.

The ${ }^{9} \mathrm{Be}$ spin alignment echo technique is described in detail elsewhere [19]. The employed pulse sequence

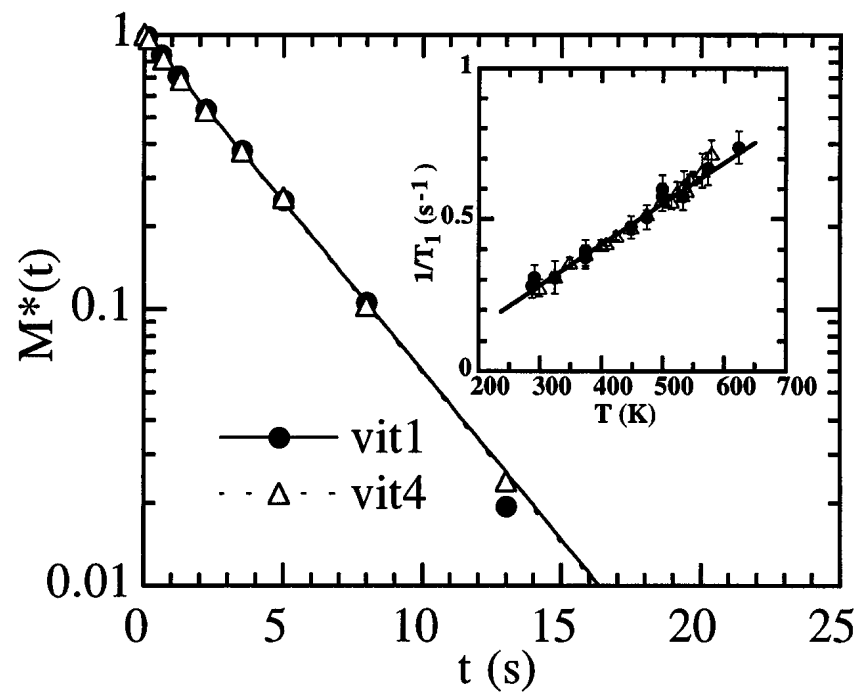

FIG. 2. The saturation recovery curves of $M^{*}(t)$ (defined in the text) vs the recovery time $t$ at $300 \mathrm{~K}$ for vit 1 and vit 4 . The decays are perfectly exponential. The inset shows $1 / T_{1}$ vs $T$ in vit1 and vit4 and the curves follow $1 / T_{1} \propto T$. is the Jeener-Broekaert sequence $90^{\circ}{ }_{x}-\tau_{1}-45^{\circ}{ }_{y}-\tau-45^{\circ}-\tau_{2}$. With a short evolution time $\tau_{1}(6 \mu \mathrm{s}$ to $15 \mu \mathrm{s}$ at $9.4 \mathrm{~T})$, the first two rf pulses create a pure quadrupole order for spin-3/2 nuclei [19]. The third pulse applied after the diffusion time $\tau$ converts the quadrupole order into an observable magnetization; an alignment echo, which arises only from the satellite transitions, is formed at $\tau_{2}=\tau_{1}$. The amplitude of the alignment echo, $S_{\text {echo }}(\tau)$, is proportional to the single-particle correlation function (SPCF) $\left\langle\sin \left(\omega \tau_{1}\right) \sin \left(\omega^{\prime} \tau_{1}\right)\right\rangle$ under the condition that $\tau>$ $T_{2}[8,19]$. The brackets represent the ensemble average over all spins. $\omega$ and $\omega^{\prime}$ are the quadrupole interactions experienced by a ${ }^{9} \mathrm{Be}$ spin during $\tau_{1}$ and $\tau_{2}$, respectively. The SPCF decays if and only if $\omega^{\prime}$ and $\omega$ are different. As discussed above, the quadrupole interaction is different from site to site in vit1 and vit4. As a result, atomic motions are expected to induce changes of $\omega$. Assuming that the motion-induced $\omega$ change in metallic glasses can be described by a Markovian process, SPCF is then proportional to $\exp (-\Omega \tau)$, where $\Omega$ is the jump frequency of atomic motions [19]. Therefore, slow motion with a jump frequency lower than $1 / T_{2}$ can be probed by measuring the alignment echo decay. The lower limit of detectable $\Omega$ is determined by the relaxation rate of the spin-state population associated with the created quadrupole order. The quadrupole order is not affected by the aforementioned slow motion. Spin-lattice relaxation mechanisms induce the quadrupole order decay with decay rate $\alpha / T_{1}$. As shown above, the ${ }^{9} \mathrm{Be}$ spin-lattice relaxation is electronic in origin; for this mechanism the decay rate is $3 / T_{1}$ [22]. Thus, the total decay rate of the alignment echo $1 / T_{\mathrm{QE}}=3 / T_{1}+\Omega$.

Short data acquisition time is crucial for conducting experiments on metallic glasses because of the tendency of crystallization near $T_{g}$. To improve the signal-to-noise ratio the pulsed spin-lock technique [23] was used in the present work. Here a train of $90^{\circ}$ pulses is applied following the Jeener-Broekaert sequence. These $90^{\circ}$ pulses separated by $2 \tau_{1}$ have the same phase as the third pulse of the Jeener-Broekaert sequence. A train of echoes with the same origin and features as the first alignment echo is detected and all the echoes are added together. With this technique, each $1 / T_{\mathrm{QE}}$ can be obtained in a few minutes. This is especially important for the structural relaxation study in which $1 / T_{\mathrm{QE}}$ is measured versus isothermal annealing time. The present study shows that the effect of isothermal annealing on Be motion is not observable in vit1 and vit4 in agreement with the EBS results [7].

Figure 3 shows the alignment echo decay versus $\tau$ at various temperatures for vit 4 . The decay curve can be fitted perfectly with a single exponential function $S_{\text {echo }}(\tau)=$ $S_{\text {echo }}(0) \exp \left(-\tau / T_{\mathrm{QE}}\right)$. The inset of Fig. 3 shows $1 / T_{\mathrm{QE}}$ vs $T$ from $300 \mathrm{~K}$ to $T_{g}$ for vit4. Below $525 \mathrm{~K}$ the relaxation caused by conduction electron spins dominates the alignment echo decay since the decay rate is proportional to $T$ and is exactly equal to $3 / T_{1}$. Above $525 \mathrm{~K}$ $1 / T_{\mathrm{QE}}$ increases rapidly, but $1 / T_{1}$ is still proportional to 


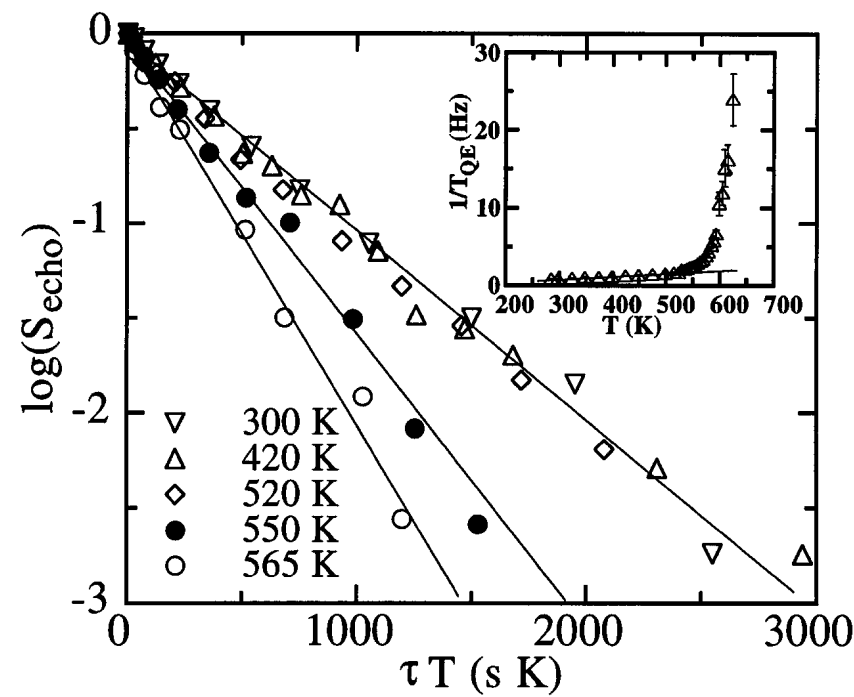

FIG. 3. The normalized alignment echo intensity $S_{\text {echo }}$ is plotted vs the diffusion time $\tau$ (scaled by $T$ ) in vit 4 at various temperatures. The corresponding curves are exponential fits. The inset shows the alignment echo decay rate $T_{\mathrm{QE}}$ vs $T$ for vit4.

$T$. Similar results were obtained for vit1. Figure 4 plots $\Omega\left(=1 / T_{\mathrm{QE}}-3 / T_{1}\right)$ vs $1 / T$ for both vit 1 and vit 4 . It exhibits an Arrhenius behavior $\Omega(T)=\Omega_{0} \exp \left(-E_{a} / k_{\mathrm{B}} T\right)$. The fittings give the same activation enthalpy $E_{a}=(1.2 \pm$ $0.15) \mathrm{eV}$ for both glasses, whereas $2 \times 10^{9}<\Omega_{0}<4 \times$ $10^{11} \mathrm{~Hz}$ for vit 1 and $1 \times 10^{10}<\Omega_{0}<2 \times 10^{12} \mathrm{~Hz}$ for vit4. Since $\mathrm{Be}$ is the smallest component in both glasses, Be motions should dominate the observed slow motions. Comparison of measured diffusion constants of $\mathrm{Be}$ and other elements in vit1 and vit 4 also supports this assumption [6]. The measured $\Omega$ is thus attributed to the Be jump frequency. The $E_{a}$ value given above is nearly identical

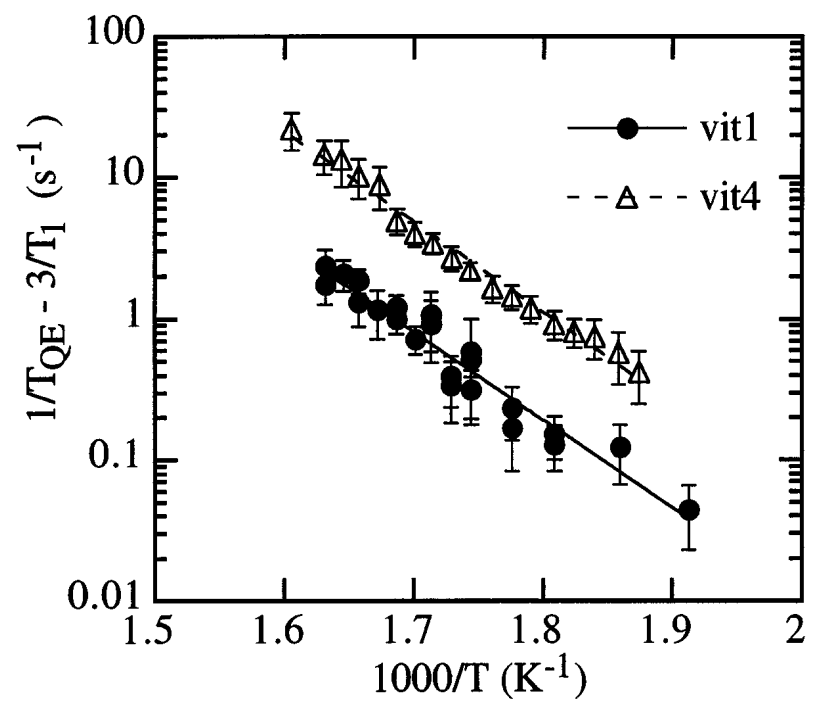

FIG. 4. $\Omega=1 / T_{\mathrm{QE}}-3 / T_{1}$ is plotted vs $1 / T$ for vit1 and vit4. The lines are Arrhenius fits with an activation enthalpy of $1.2 \mathrm{eV}$. to that determined by EBS [7]. The difference in $\Omega(T)$ between vit1 and vit4 is also consistent with the EBS results [7]. These similarities with the EBS data imply that the NMR-detected motions are due to Be diffusion and are not mainly due to motions of other atoms. Theoretical models suggest that there is a large distribution of energy barriers in glassy systems. For long-range displacement the high energy barriers provide the main hindrance to diffusion whereas for short-range displacement, atoms would most likely jump over the low energy barriers [1]. Thus, it is somewhat surprising that NMR and EBS give the same activation enthalpy.

Several diffusion mechanisms have been proposed for diffusion in metallic glasses [1-3]. One of these is the vacancy-assisted diffusion mechanism [3]. Since Be is expected to be the fastest moving atoms in vit1 and vit4, a vacancy jump is expected to be correlated with the jump of neighboring Be atoms. The vacancy concentration in vit1 and vit4 is expected to be low. The measured free volume in vit 1 is about $0.1 \%$ [24]. Therefore, there exists a distribution of distances between $\mathrm{Be}$ atoms and vacancies. Given a time interval $\tau$, there should be a distribution of Be jump probability at sufficiently low temperatures under vacancy-assisted diffusion; a Be atom initially (at the beginning of $\tau$ ) close to a vacancy will have a higher probability to execute a jump than those initially far from vacancies. In this case, the alignment echo decay is described by

$$
S_{\text {echo }}(\tau)=\left[\sum_{i} A\left(w_{i}\right) \exp \left(-w_{i} \tau\right)\right] \exp \left[-\left(3 / T_{1}\right) \tau\right]
$$

where $w_{i}$ is one of the jump rates of Be and is smaller than the jump rate of a vacancy. $A\left(w_{i}\right)$ is the distribution of $w_{i}$. As shown in Fig. 3, the ${ }^{9} \mathrm{Be}$ alignment echo decay follows a single exponential function within the entire temperature range. This suggests that the Be diffusion mechanism is spatially homogeneous and is not consistent with vacancyassisted diffusion. We should keep in mind, however, that if the observed signal by NMR originates only from the Be atoms which are far away from the vacancies, the expected inhomogeneity would not show up. However, the expected small vacancy concentration and the observed correct central to satellite transition intensity ratio indicate that the majority of the ${ }^{9} \mathrm{Be}$ spins are observed by NMR.

For a direct diffusion process the Be jump rate $\Omega$ can be written as [1]

$$
\Omega=Z \nu_{0} \exp \left(S_{m} / k_{\mathrm{B}}\right) \exp \left(-H_{m} / k_{\mathrm{B}} T\right),
$$

where $H_{m}$ and $S_{m}$ are the migration enthalpy and entropy, respectively, and $\nu_{0}$ is the attempt frequency which is comparable to the Debye frequency of about $10^{13} \mathrm{~Hz}$. $Z$ is a constant representing, for instance, the number of nearest interstitial sites for the interstitial mechanism. Using the measured prefactor values of $\Omega_{0} \equiv Z \nu_{0} \exp \left(S_{m} / k_{\mathrm{B}}\right)$, this implies that $-8.5<S_{m} / k_{\mathrm{B}}<-3.2$ for vit1 and $-6.9<$ $S_{m} / k_{\mathrm{B}}<-1.6$ for vit4. This excludes the conventional interstitial mechanism for which $S_{m} / k_{\mathrm{B}}=1$ is expected. 
Since vit 1 and vit4 consist of small $(\mathrm{Be})$, medium $(\mathrm{Cu}$ and $\mathrm{Ni}$ ), and large ( $\mathrm{Zr}$ and $\mathrm{Ti}$ ) atoms, it favors dense atomic packing. This might be effective in reducing interstitial diffusion. Similarly, the measured $\Omega_{0}$ values are too small to be compatible with thermally generated vacancyassisted diffusion.

The observed effective negative migration entropy and the homogeneous nature of the diffusion mechanism favor the spread-out free volume mechanism [25]. The mobility of such free volume is too low to have a direct effect on Be diffusion below $T_{g}$. However, thermal fluctuations of spread-out free volume could lead to the temporary formation of a volume larger than a critical volume $v^{*}$; Be hopping into such temporarily formed volume leads to Be diffusion. The probability of finding a volume fluctuation near a given atom larger than $v^{*}$ is given by $\exp \left(-\gamma \boldsymbol{v}^{*} / \boldsymbol{v}_{f}\right)$ which is the same for all Be atoms [25]. Here $\gamma$ is a geometric factor, and $v_{f}$ is the average free volume of an atom. Clearly, this mechanism is consistent with the observed pure exponential decay of $S_{\text {echo }}(\tau)$. Furthermore, the factor $\exp \left(-\gamma v^{*} / v_{f}\right)$ also explains the observed negative effective migration entropy [1]. The faster atomic motions in vit4 are thus a result of a larger $v_{f}$ in vit4 than in vit1.

In contrast to previous local relaxation measurements using magnetic after effect and induced magnetic anisotropy techniques [1], NMR measurements of local Be motion give the same activation enthalpy as EBS diffusion measurement. The lack of activation enthalpy distribution is consistent with the negative values of $S_{m} / k_{\mathrm{B}}$ which indicates that the thermal fluctuation of spread-out free volume involves a group of atoms. Thus, the activation enthalpy distribution is averaged out for Be motion. This result is also consistent with the pure exponential decay of $S_{\text {echo }}(\tau)$.

In summary, the ${ }^{9} \mathrm{Be}$ alignment echo technique provides detailed information on the characteristics of $\mathrm{Be}$ diffusion in vit1 and vit4. The result is consistent with the direct diffusion process for Be involving thermal fluctuations of spread-out free volume. No distribution of activation enthalpy is visible through alignment echo decay. This is consistent with the fact that the NMR-detected activation enthalpy of $1.2 \mathrm{eV}$ for local motion is the same as that of long-range atomic motions determined by EBS.

We thank Dr. A. Kleinhammes and L.P. Potter for experimental assistance. This work was supported by the U.S. Army Research Office under Contract No. DAAH04-96-1-0185, the National Science Foundation under Contract No. DMR-9802101, and the
Department of Energy under Contract No. DEFG-0386ER45242.

*Electronic address: yuewu@physics.unc.edu

[1] H. Kronmüller, W. Frank, and A. Hörner, Mater. Sci. Eng. A 133, 410 (1991); W. Frank, J. Horvath, and H. Kronmüller, ibid. 97, 415 (1988).

[2] F. Faupel, Phys. Status Solidi (a) 134, 9 (1992); P. Klughist, K. Rätzke, S. Rehders, P. Troche, and F. Faupel, Phys. Rev. Lett. 80, 3288 (1998).

[3] A. Grandjean and Y. Limoge, Acta Mater. 45, 1585 (1996).

[4] H. J. Höfler, R. S. Averback, G. Rummel, and H. Mehrer, Philos. Mag. Lett. 66, 301 (1992).

[5] S. K. Sharma, M.-P. Macht, and V. Naundorf, Phys. Rev. B 49, 6655 (1994).

[6] E. Budke, P. Fielitz, M.-P. Macht, V. Naundorf, and G. Frohberg, Defect Diffus. Forum 143-147, 825 (1997).

[7] U. Geyer, S. Schneider, W. L. Johnson, Y. Qiu, T. A. Tombrello, and M.-P. Macht, Phys. Rev. Lett. 75, 2364 (1995); U. Geyer, W. L. Johnson, S. Schneider, Y. Qiu, T. A. Tombrello, and M.-P. Macht, Appl. Phys. Lett. 69, 2492 (1996).

[8] H. W. Spiess, J. Chem. Phys. 72, 6755 (1980).

[9] G. Fleischer and F. Fujara, NMR Basic Princ. Prog. 30, 159 (1994).

[10] K. Schmidt-Rohr and H. W. Spiess, in Multidimensional Solid-State NMR and Polymers (Academic Press, London, 1994).

[11] I. Farnan and J. F. Stebbins, Science 265, 1206 (1994).

[12] H. Eckert, Curr. Opin. Solid State Mater. Sci. 1, 465 (1996).

[13] A. Seeger, D. Wolf, and H. Mehrer, Phys. Status Solidi (b) 48, 481 (1971).

[14] D. C. Ailion, Adv. Magn. Reson. 5, 177 (1971).

[15] Y. Chabre, J. Phys. F 4, 626 (1974).

[16] L. Bottyán, D. L. Beke, and K. Tompa, Phys. Status Solidi (b) 118, 835 (1983).

[17] A. Peker and W. L. Johnson, Appl. Phys. Lett. 63, 2342 (1993).

[18] R. Busch, E. Bakke, and W. L. Johnson, Mater. Sci. Forum 235-238, 327 (1997).

[19] X.-P. Tang and Y. Wu, J. Magn. Reson. 133, 155 (1998).

[20] D. E. Barnaal, R. G. Barnels, B. R. McCart, L. W. Mohn, and D. R. Torgeson, Phys. Rev. 157, 510 (1967).

[21] J. Winter, in Magnetic Resonance in Metals (Clarendon, Oxford, 1971).

[22] E. R. Andrew and D. P. Tunstall, Proc. Phys. Soc. London 78, 1 (1961).

[23] J. R. C. van der Maarel, J. Chem. Phys. 94, 4746 (1990).

[24] A. Masuhr, T. A. Waniuk, R. Busch, and W. L. Johnson (to be published).

[25] F. Spaepen, Acta Metall. 25, 407 (1977). 